http://jmscr.igmpublication.org/home/ ISSN (e)-2347-176x ISSN (p) 2455-0450 crossref DOI: https://dx.doi.org/10.18535/jmscr/v7i8.131

\title{
Anaesthesia approach to a six month old child of Meningomyelocele with Hydrocephalus and Arnold Chiari Malformation
}

\author{
Authors \\ Dr Kothare Pratima, MD, DNB Anaesthesia ${ }^{1}$, Dr Daga Khushboo, MD Anaesthesia ${ }^{2}$ \\ ${ }^{1}$ Consultant Anaesthesiologist, Assistant Professor Bombay, Hospital Institute of Medical Sciences, Mumbai \\ ${ }^{2}$ Resident, Bombay Hospital Institute of Medical Sciences, Mumbai
}

*Corresponding Author

Dr Daga Khushboo, MD Anaesthesia

Resident, Bombay Hospital, Institute of Medical Sciences, Mumbai, India

\begin{abstract}
MeningoMyeloCele (MMC) is a complex congenital spinal anomaly resulting from neural tube defect during first 4 weeks of gestation. Early surgery within 48 hours of birth should be performed to prevent rupture, infection, tethering and its sequelae.

Approach to anesthesia is multifold, proper position to protect the rupture of thin walled sac when supine, and position to secure the airway and avoid compression of cervical cord due to ACM, intraoperative blood loss and temperature management.

We report a case of 6 month, $7 \mathrm{~kg}$ child of MMC with ACM with hydrocephalus and bilateral congenital CTEV with a head circumference of $49 \mathrm{cms}$, and an enlarging and discharging MMC sac.

Keywords: MMC, 6 months, hydrocephalus, ACM, Position, Venous access, Hypothermia.
\end{abstract}

\section{Introduction}

Embryogenic neural tube fusion occurs during the first 4 weeks of gestation. Failure of fusion causes herniation of meninges with or without neural element [Meningomyelocele -MMC /Meningo cele $]^{1,2}$

Folic acid helps to prevent neural tube defects when given from first trimester itself or even before planning a pregnancy.

Arnold Chiari Malformation $[\mathrm{ACM}]$ and hydrocephalus are generally associated abnormalities with MMC. Congenital conditions like club foot (CTEV), extrophy of bladder prolapsed uterus, tracheoesophageal fistula,
Klippel Feil syndrome and rarely cardiac defects are other associated congenital abnormalities. ${ }^{3}$

The clinical presentation will vary according to anatomical defect involved. Orthopedic and urologic symptoms may occur with uncorrected meningocele as a result of tethering of spinal cord by sacral nerve root $^{4}$

ACM is a downward displacement of the cerebellar vermin into upper cervical spinal canal and elongation of brain stem and $4^{\text {th }}$ ventricle. Therefore when ACM is present with MMC and hydrocephalus, it requires special positioning during intubation, and also there should be no pressure on the exposed neural placode and a postoperative watch for stridor and apnoea. ${ }^{5}$ 
Therefore, anaesthesia administration and management in MMC is challenging, concerns being pediatric age group, airway management positioning, associated systemic co morbidities and post operative recovery after repair ${ }^{6}$

An ideal approach to a MMC child is to operate within $24 \mathrm{hrs}$ of birth to reduce the sequelae of MMC, close the defect; place a ventriculoperitoneal shunt or delay placing the shunt till hydrocephalus sets in.

\section{Material and Methods}

A 6 month old female child weighing $6.9 \mathrm{kgs}$ (birth weight $=3.5 \mathrm{kgs}$ ) presented with swelling in lower back, which was size of lemon at birth and gradually increased, with some clear fluid discharge from the swelling .Parents also noticed deformed feet with very less/no activity in lower limbs, upper limbs moving normally.

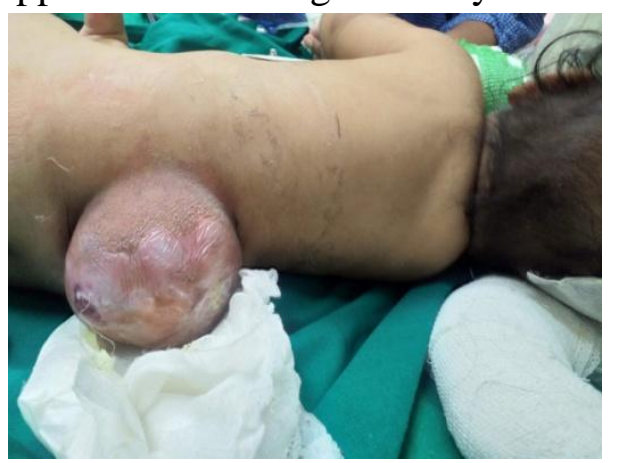

Figure a

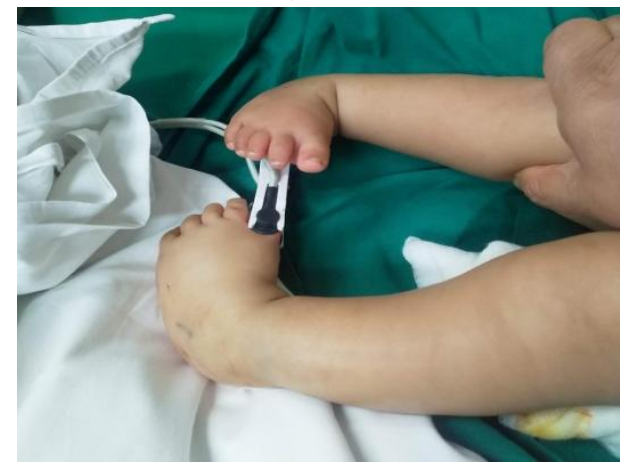

Figure $b$

No breathing difficulty, urinary or stool complaints.

Although swelling was diagnosed on USG at 7 months of gestation child presented to us 6 months after birth.

\section{On examination}

Swelling in lumbar region of about $8-10 \mathrm{cms}$. Both lower limbs no tone. Power in lower limbs $1 / 5$, flicking movements of toes, $0 / 5$ at ankle and knee, $1 / 5$ at hip. Truncal power $3 / 5$. Muscle nutrition normal. Plantar reflex no response. B/L CTEV. Head circumference of $49 \mathrm{~cm}$.

MRI findings as shown in figure (figure c)

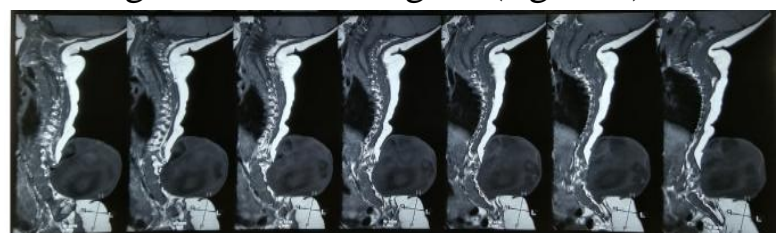

Figure c

On investigation, Haemoglobin 11;6, hyponatremia $-113 \mathrm{meq} / \mathrm{lts}$ which was corrected with $3 \% \mathrm{NaCl}$ at $3.6 \mathrm{ml} / \mathrm{hr}$ over $24 \mathrm{hrs}$. albumin $=12.8$. All other investigations were WNL.

Operation proposed right ventriculoperitoneal shunt in supine and repair of MMC in prone position in the same sitting.

Difficult intubation cart was prepared in anticipation. (Figure d)

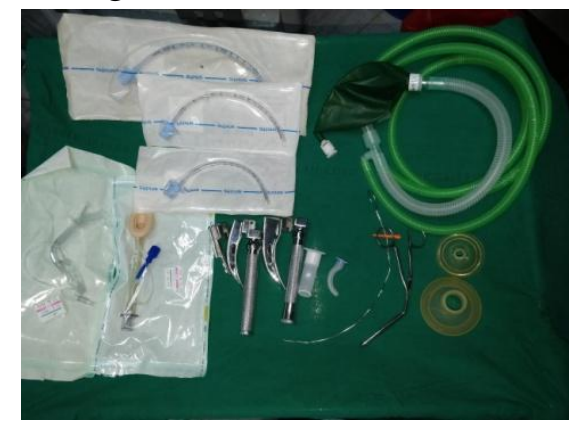

Figure d

Routine monitoring SPO2, ECG, NIBP connected Special Monitoring: Arterial blood gas , serum electrolytes blood sugar, core rectal temperature, urine output

Intravenous access with 24 no. angiocath on left anterior aspect of wrist under $\mathrm{O} 2$ and sevoflurane mask. No other visible peripheral veins in both UL or LL.

Position for mask ventilation and intubation- in supine position with doughnut ring under the lumbar MMC and folded towel under the trunk with two donuts under the head for proper airway alignment (Figure e) 


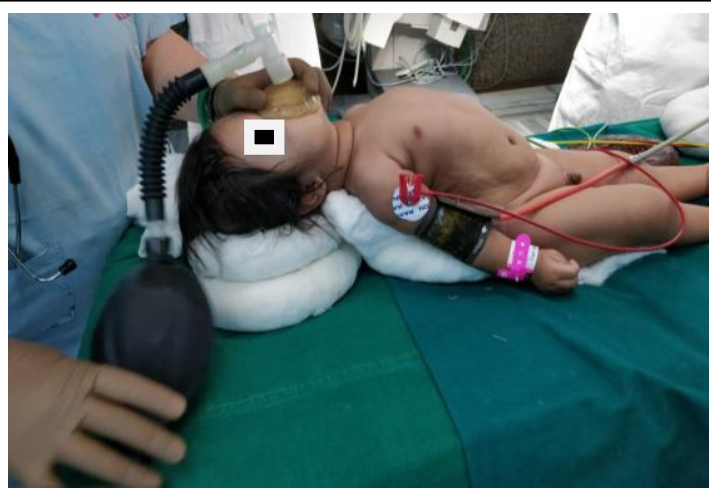

Figure e

Preoxygenated, premedicated with atropine $0.01 \mathrm{mg} / \mathrm{kg}$, induced with thiopentone $4 \mathrm{mg} / \mathrm{kg}$ and vecuronium $0.1 \mathrm{mg} / \mathrm{kg}$. Intubate with 3.5 cuff tube -low volume low pressure secured, throat packed and connected to close circuit ventilator with TV of $8 \mathrm{ml} / \mathrm{kg}$ and $\mathrm{RR}$ of $18 / \mathrm{min}$.

Due to fragile and inadequate peripheral venous access, left internal jugular venous access attempted under ultrasound guidance but it was unsuccessful despite multiple attempts. Subsequently ultrasound guided left subclavian cannulation performed.

IV fluids Isolyte $\mathrm{P}$ started through infusion pump at the rate of $6 \mathrm{ml} / \mathrm{kg} / \mathrm{hr}$. Blood replaced as per blood loss. VP shunt completed uneventfully in 30 minutes.

There after prone position given for MMC repair, with proper size bolster under iliac crest and shoulder, keeping abdomen free for ventilation and prevention of venous congestion with proper pressure points care (figure f)

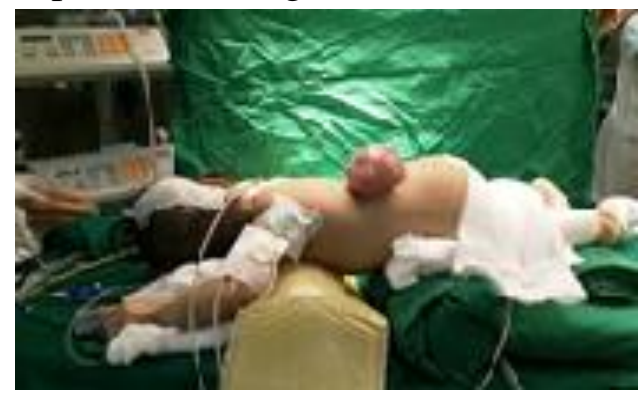

Figure $\mathrm{f}$

Special care taken to keep the child warm with warm IV fluid and irrigating fluids, warmer blanket and wrapping of limbs with cotton.

Maintenance with gas oxygen, nitrous, sevoflurane and intermittent vecuronium.
Reversal was standard with atropine and neostigmine, after adequate spontaneous respiration, eye opening and upper limb movements. However there was a delay in emergence of about forty five minutes due to hypothermia. (34 degrees at the time of reversal) despite proper precautions taken.

For Postoperative pain relief, IV paracetamol and paediatric diclofenac suppository inserted, thereafter shifted to PICU for further management.

\section{Discussion}

MMC with an incident of 0.4 to 1 per 1000 live births is one the common malformations of CNS, with a leading cause of paralysis and lifelong disabilities including paraplegia, hydrocephalus, chiari malformation, incontinence, skeletal deformities and mental impairment.

Folic acid should be supplemented ideally before conception or as soon as pregnancy is confirmed, to prevent neural tube defects.

The timing of surgery is usually in the first $48 \mathrm{hrs}$ after birth, to prevent rupture of sac, infection and neurological complications ${ }^{7}$

About 1/3 rd of babies develop symptomatic chiari malformation where in cerebellar tonsils are displaced below the foramen magnum with elongation and compression of the brainstem and obliteration of cisterna magna, hence developing symptoms like inspiratory stridor, apnoea, swallowing dysfunction, bradycardia etc. This important associated abnormality gives us the indication for a very careful positioning during induction of anaesthesia, therefore avoid extension during laryngoscopy as it would lead to brainstem compression and ventilator dependence. 80-90\% of MMC children have associated hydrocephalus, due to any contributory factor like aqueduct stenosis, $4^{\text {th }}$ ventricle outlet obstruction, obliteration of posterior fossa, sub arachnoid space etc. If hydrocephalus is significant with a large head circumference, it poses a difficulty in airway management and again special care is 
required to position the head for mask ventilation and intubation. ${ }^{8}$

Presence of other skeletal deformities like CTEV or scoliosis adds to the challenges.

Central venous access is an option to be considered as MMC repair requires blood replacement and IV fluids for a longer duration, especially with very fragile or no peripheral venous access in infants. Intravenous access on the lower limbs may not be possible in CTEV deformity. Intra arterial cannulation can be considered for Blood pressure and blood gas analysis.

Hypothermia in infants occur quite commonly under general anaesthesia, as it depresses the thermoregulatory response and autonomic control below defect is abnormal. Heat is lost from the core to the cooler peripheral tissue. This is compounded by the cold operating room. Thus prolonged hypothermia may lead to acidosis and impaired tissue perfusion, which thus lead to delayed awakening, cardiac irritability, respiratory depression, increased pulmonary vascular resistance and altered drug responses ${ }^{9}$.Thus prevention of hypothermia at the outset is important by very simple measures like adjusting the operative room temperature, wrapping of all extremities and head with cotton blankets, warm intravenous and irrigating fluids and inhaled anesthetics to be given through humidifier.

Serum electrolyte and blood sugar monitoring are important for a better recovery from anesthesia

Positioning of child for bag mask ventilation and intubation and similar precautions during extubation is a very important aspect of airway alignment management in MMC especially with associated ACM.

Reversal and recovery is equally crucial here. Extubation to be done only when the child is awake and breathing well. The criteria for extubation are intact cough and gag reflex, a forced vital capacity in excess of $10 \mathrm{ml} / \mathrm{kg}$, an air leak around the tube, maintenance of oxygen saturation on spontaneous breathing, adequate reversal of neuromuscular blockade shown by sustained arm lift and spontaneous breathing.

Possible causes of delayed recovery are hypothermia, inadequate reversal, electrolyte imbalance, prolonged effect of anesthetic agents ${ }^{10}$ Post operative ventilator support is rarely required in pure MMC repair However associated ACM or any intra operative complications may demand respiratory support.

Post operative pain relief to be addressed as per standard institutional protocol.

\section{Conclusion}

Prevention of rupture of MMC sac requires foremost attention hence special position required to be customized to cushion the sac. This further involves proper alignment and positioning for bag mask ventilation and intubation. Presence of Arnold Chiari Malformation poses a challenge during intubation and a risk of stridor /apnoea in the post-operative recovery.

Paediatric airway management, intravenous access, correction of intra operative fluid and electrolyte imbalance, maintaining proper core temperature is a key to successful outcome of MMC repair.

Conflict of interest - Nil

Source of Funding- self

\section{References}

1. Charles lee, Igor luginbuehl, Bruno bissonnette, Linda j.mason. pediatric diseases in: Roberta L.Hines, Catherine marshall, editor. Stoelting „s Anaesthesia and coexisting diseases 5 th edition. Elsevier Churchill livingstone; 2008 pg: 608- 609

2. Charles J.cote. Pediatric anesthesia in: Ronald D Miller, editor. Millers anesthesia 6th edition; Elsevier Churchill livingstone; 2005, pg2395..

3. John HM, Haervey BS, Laura FS. Malformation of the central nervous system. J. Child neurology 2006 ;285-366 
4. Rinaldi F, Cioffi FA, Columbano L, Krasagakis G, Bernini FP, Tethered cord syndrome. Journal of neurosurgical science 2005; 49: 131-5

5. Soriano SG, McManu ML. Pediatric neuroanesthesia and critical care. In: Cottrell JE, Young WL, editors. Cottrell and Young's Neuroanesthesia. Philadelphia: Mosby Elsevier; 2010. pp. 327-42.

6. Singh D, Rath GP, Dash HH, Bithal PK. Anesthetic concerns and perioperative complications in repair of myelomeningocele: A retrospective review of 135 cases. J Neurosurg Anesthesiol. 2010;22:11-5.

7. Herman JM, McloneStors BB, Dauser RC. Analysis of 153 patients with myelomeningocele or spinal lipoma are operated upon for a tethered cord : presentation, management and outcome. Pediatric neurosurgery 1998;19:243-249327

8. Oren J, Kelly DH, Todres ID. Respiratory complications in pateitns with myelodysplasia and Arnold Chiari malformation. Am. J. Dischild 1986; 140:221-224.

9. Elliot J. Krane, Bridget M. Philip, Kelly K.yeh, Karen B.Domino, Anesthesia for pediatric neurosurgery in: Etsurok. Motoyamma and Peter J.Davis, editors: Smith"esanaesthesia for infants and children, 7 th edition: mosby: 2005.

10. Chand MB ,Agarwal J , Dr P.Bista. Anaesthetic challeneges and management of myelomeningocele repair.PMJN(Post graduate medical journal of NAMS) Volume 11, Number 1, Jan-June 2011. 